\title{
Sexual Dysfunction, Gender Dysphoria, Self-Control Disorders
}

This column includes questions concerning sexual dysfunctions, gender dysphoria, and disruptive, impulse-control, and conduct disorders. A number of changes occurred in DSM-5 concerning these disorders. In contrast to $D S M-I V$, gender-specific sexual dysfunctions have been added and, for females, sexual desire and arousal disorders have been combined into one disorder (female sexual interest/arousal disorder). Gender dysphoria is new in DSM-5 and reflects a change in conceptualization of its defining features by emphasizing the phenomenon of "gender incongruence" rather than cross-gender identification per se, as was the case in DSM-IV gender identity disorder. The chapter on disruptive, impulse control, and conduct disorders is new to DSM5. It combines disorders that were previously included among disorders usually first diagnosed in infancy, childhood, adolescence, or impulsecontrol disorders not otherwise specified (that is, oppositional defiant disorder; conduct disorder; other specified and unspecified; disruptive, impulse-control, conduct disorders; and intermittent explosive disorder). These disorders are all characterized by problems in emotional and behavioral self-control.

The questions below are from DSM-5 Self-Exam Questions: Test Questions for the Diagnostic Criteria, which will be available in August. It may be preordered from American Psychiatric Publishing at http:// www.appi.org/SearchCenter/Pages/ SearchDetail. aspx? ItemId $=62467$.

The questions were developed under the leadership of Philip Muskin, M.D., a professor of clinical psychiatry at Columbia University

College of Physicians and Surgeons.

The book, available in August, contains 500 questions for all the cat- egories of psychiatric disorders and includes Section III.

1. Sexual Dysfunction: Which of these describes a condition or disorder, which would be properly diagnosed as Other Specified Sexual Dysfunction?

a) Medical-induced sexual dysfunction
b) Sexual aversion
c) Erectile dysfunction
d) Female sexual interest/arousal disorder
e) Delayed ejaculation

\section{Answer: B. Sexual aversion}

Rationale: All of the answers except Sexual Aversion refer to diagnosable disorders in the Sexual Dysfunction category, each with its own specific set of criteria. Sexual Aversion, described as "extreme aversion to and avoidance of genital sexual contact with a sexual partner," is given as an example of a presentation that is not currently recognized formally as a disorder, and would therefore be diagnosed as "Other Specified Sexual Dysfunction, Sexual Aversion."

2. Gender Dysphoria: Which of the following diagnoses has been eliminated in DSM-5?

a) Gender identity disorder

b) Borderline personality disorder

c) Gender dysphoria

d) Pedophilic disorder

e) Transvestic disorder

\section{Answer: A. Gender identity disor- der}

Rationale: A major change in DSM-5 is that the diagnosis of gender identity disorder (GID) has been eliminated. There is no consensus in the field about when or whether atypical gender identities represent a disorder. DSM-5 focuses on gender dysphoria (which replaces both the former GID and gender incongruence). This term emphasizes dysphoria as the clinical problem, rather than on either identity or incongruence, and is therefore more descriptive. The concept of gender dysphoria is also no longer dichotomized between male and female, allowing for an infinite range of gender experience.

3. Disruptive, Impulse-Control, and Conduct Disorders: A 16-yearold boy with a long history of defiant behavior toward authority figures also has associated aggression toward peers (gets into fights at school), his parents, and objects (punching holes in walls, breaking doors). Furthermore, he has begun to steal from stores and his parents (money, jewelry), and he frequently lies. He does not seem pervasively irritable or depressed, and has no sleep disturbance or psychotic symptoms. What is the likely DSM-5 diagnosis for this patient?

a) Oppositional defiant disorder

b) Conduct disorder

c) Attention-deficit/hyperactivity disorder

d) Major depressive disorder

e) Disruptive mood dysregulation disorder

\section{Answer: B. Conduct disorder}

Rationale: In this example, the boy displays aggression toward people, destruction of property, and deceitfulness or theft (all part of Criterion A for Conduct disorder in DSM-5). Individuals with oppositional defiant disorder (ODD) are not typically aggressive toward people or animals, and they do not generally destroy property or exhibit patterns of behavior involving theft or deceit. In addition, individuals with ODD 
a have problems with emotional dysregulation as a more prominent and pervasive feature of their presenta-

tion. There is not enough information

from the vignette to establish a

diagnosis of ADHD, and the lack of a pervasive mood disturbance argues against a diagnosis of major depressive disorder or disruptive mood dysregulation disorder. 